\title{
Marijuana and head and neck cancer: an epidemiological review
}

\author{
Michael Xie ${ }^{1,2}$, Michael K. Gupta', Stuart D. Archibald ${ }^{2}$, B. Stanley Jackson', James Edward Massey Young ${ }^{1}$ and \\ Han Zhang ${ }^{1 *}$
}

\begin{abstract}
Background: Marijuana is the most widely used illicit substance in Canada. To date, no conclusive study has looked at the epidemiologic basis of marijuana use and head and neck cancer (HNC). Due to the imminent recreational legalization of marijuana in Canada, the epidemiologic relationship between marijuana use and HNC is becoming increasingly important.
\end{abstract}

Objective: To examine the epidemiologic characteristics of HNC patients who are recreational marijuana users.

Methods: This study was conducted at a single tertiary care centre from 2011 to 2014. Patients were enrolled consecutively at time of diagnosis of malignancy. Data was prospectively collected and included socioeconomic factors, alcohol/tobacco history, tumor characteristics, and treatment modality. Marijuana use was defined as current usage on an at least weekly basis.

Results: Eight hundred seventy-nine patients met inclusion and exclusion criteria. Seventy-four (8.4\%) patients were classified as marijuana users. Compared to non-users, marijuana users were less likely to be married $(p=0.048)$ and had less significant tobacco smoking history $(p=0.004)$. There were no significant differences between other socioeconomic factors or local and regional disease $(p>0.05)$. Marijuana users differed in the proportion of cancers stratified by primary site $(p<0.0001)$, with higher rates of $p 16+$ oropharyngeal cancers, and treatment modality $(p<0$. 0001), with more use of chemoradiation.

Conclusions: HNC patients who were marijuana users were less likely to be married and smoke tobacco. They have a distinct cancer site prevalence and are more likely to be treated by chemoradiation. Understanding the epidemiological breakdown of marijuana users amongst HNC patients will be a useful adjunct for future studies.

Keywords: Marijuana, Head and neck cancer, Cancer epidemiology

\section{Introduction}

Cannabis sativa, otherwise known as marijuana, is the most commonly used illicit drug in Canada. The 2012 Canadian Community Health Survey-Mental Health found that 3.4 million (12.2\%) Canadians aged 15 or older reported using marijuana in the past year and the prevalence of lifetime marijuana use was determined to be as high as $42.5 \%$ [1]. Canada's trend towards recreational legalization has been providing impetus to better

\footnotetext{
* Correspondence: hanzhang@stjosham.on.ca

Presentation: This project was presented by Michael Xie at the $72^{\text {nd }}$ Annual

Meeting of the Canadian Society of Otolaryngology at Quebec City, Quebec on June $16^{\text {th }}-19^{\text {th }} 2018$.

'Division of Otolaryngology-Head and Neck Surgery, McMaster University, G811, 50 Charlton Avenue E, Hamilton, ON L8N 4A6, Canada

Full list of author information is available at the end of the article
}

elucidate the association between marijuana and head and neck cancer (HNC). Regardless of the change in usage with recreational legalization, a sizable percentage of the Canadian population might have the potential to increase marijuana usage based on current statistics [13]. Despite this, there is a tangible gap within the current literature in understanding the epidemiological variations of HNC patients in Canada who are marijuana users compared to those who are not.

Bridging this gap in knowledge and understanding is important to better explore the relationship between marijuana and HNC. HNC patients, much like others who suffer from life altering oncology diagnoses, often report impacts on their quality of life secondary to psychiatric and physical symptoms in relation to diagnosis

(C) The Author(s). 2018 Open Access This article is distributed under the terms of the Creative Commons Attribution 4.0 International License (http://creativecommons.org/licenses/by/4.0/), which permits unrestricted use, distribution, and 
and treatment [4-8]. Marijuana has been proposed to have a potential therapeutic role in in these patients due to its ability to enhance relaxation, decrease stress, and improve quality of life; however there is a lack of direct evidence at this time $[1,9,10]$. Other than the potential mood effects of marijuana, its role as a carcinogen is not yet established as the literature has been inconclusive in finding a link between marijuana as a cause for HNCs [11-14]. Although specifics of such relationships between marijuana and HNC are beyond the scope of this current study, a better understanding of the epidemiology of marijuana use in HNC can assist future work in these areas of research.

It is therefore, the aim of this study to examine the epidemiological variations within patients who are marijuana smokers diagnosed with HNCs.

\section{Methods}

This study received ethics approval from the Hamilton Integrated Research Ethics Board.

\section{Data collection}

Consecutive patients were recruited prospectively and consecutively at the time of biopsy proven diagnosis of HNC from the Juravinski Cancer Center, Hamilton, Ontario from July 2011 to July 2014. All patient data was collected prospectively. Patients were included if they were greater than 17 years old and had a pathologically confirmed diagnosis of cancer of the head and neck. Patients were excluded if they had incomplete data sets.

Included patients completed a structured questionnaire (Additional file 1) that requested information on the following variables: marijuana use, education level, ethnicity, marital status, alcohol consumption, tobacco use, pack year history, income, employment status, age, gender, Karnofsky score. Diagnostic and treatment data were also collected and included: primary site of cancer, clinical $\mathrm{T}$ stage, clinical $\mathrm{N}$ stage, and modality of treatment. For oropharyngeal cancers, p16 status was also collected. Socioeconomic data was cross-referenced with Canada Census Data from years, 2011 and 2015. Cases were linked to income quintiles using patients' postal codes.

\section{Statistical analysis}

Patients were split into marijuana user and non-marijuana user groups based on a history of marijuana usage. Marijuana use was defined as current usage on an at least weekly basis. This is based on previous studies that have examined the association of marijuana use and HNC
$[15,16]$. All patients within the marijuana user group were using marijuana at the time of the study collection.

Descriptive statistics were used to compare patient demographics between marijuana smokers and non-smokers. Discrete variables were compared using Chi-squared or fischer's exact probability tests. $P$-values less than 0.05 were considered statistically significant. All analyses were performed using SPSS 20.0 (SPSS Inc., Chicago, IL.).

\section{Results}

Eight hundred seventy-nine total patients met inclusion and exclusion criteria. 74 (8.4\%) patients in the study population were classified as marijuana users. 805 (91.6\%) patients were classified as non-marijuana users.

Table 1 summarizes patient characteristics for the two groups. The marijuana group had a mean age of 62.26, which was not statistically significantly different to the mean age of 56.76 in the non-marijuana group $(p=0.068)$. There was a higher proportion of male patients within the marijuana group compared to the non-marijuana group, $85.1 \%$ vs $71.2 \%$ respectively $(p=0.011)$. The predominant ethnicity in both groups was Caucasian, 97.3 and $95.1 \%$ in

Table 1 Patient Characteristics

\begin{tabular}{|c|c|c|c|}
\hline Variable & Non-Marijuana & Marijuana & $\overline{p \text {-Value }}$ \\
\hline $\mathrm{N}$ & 805 & 74 & \\
\hline Mean Age & 56.76 & 62.26 & 0.068 \\
\hline Gender & & & 0.011 \\
\hline Male & $573(71.2)$ & $63(85.1)$ & \\
\hline Female & $232(28.8)$ & $11(14.9)$ & \\
\hline Ethnicity & & & 0.537 \\
\hline Caucasian & $766(95.1)$ & $72(97.3)$ & \\
\hline Black & $7(0.9)$ & $1(1.4)$ & \\
\hline Asian/Indian Descent & $19(2.4)$ & $0(0)$ & \\
\hline Latin Descent & $8(1.0)$ & $1(1.4)$ & \\
\hline Native/Aboriginal & $5(0.6)$ & $0(0)$ & \\
\hline Alcohol Use & & & 0.183 \\
\hline None & $279(34.6)$ & $23(31.1)$ & \\
\hline$<5$ Drinks/Week & $381(47.3)$ & $24(32.4)$ & \\
\hline 5-20 Drinks/Week & $89(11.0)$ & $13(17.6)$ & \\
\hline > 20 Drinks/Week & $65(8.1)$ & $14(18.9)$ & \\
\hline Tobacco Use & & & 0.001 \\
\hline Yes & $439(54.5)$ & $20(27.0)$ & \\
\hline No & $366(45.5)$ & $54(73.0)$ & \\
\hline Tobacco Pack Year History & & & 0.004 \\
\hline$<20$ & $526(65.4)$ & $58(78.4)$ & \\
\hline$>21$ & $279(34.6)$ & $16(21.6)$ & \\
\hline Karnofsky Score & 87.0 & 92.4 & 0.084 \\
\hline
\end{tabular}


the marijuana and non-marijuana groups, respectively $(p=0.537)$. Overall, marijuana and non-marijuana patients did not differ in alcohol use $(p=0.183)$, but marijuana patients trended towards heavier drinking with more patients reporting 5-20 and > 20 drinks per week $(17.6 \%$ vs 11.0 and $18.9 \%$ vs $8.1 \%$, respectively). Tobacco use also differed between the two groups. A smaller proportion of patients in the marijuana group had tobacco usage compared to the non-marijuana user group $(27.0 \%$ vs $54.5 \%, p=0.001)$. Additionally, fewer marijuana patients reported having a significant smoking history (greater than 21 pack-years) compared to the non-marijuana user group $(21.6 \%$ vs $34.6 \%, p=0.004)$. Marijuana users reported a marginally higher Karnofsky score $(92.4 \%$ vs $87.0 \%)$ as compared to the non-marijuana user group, but this difference in quality of life was not statistically significant $(p=0.084)$.

Socioeconomic characteristics are summarized in Table 2 and statistical analysis represents comparison of proportions between the groups. Patients in both groups reported similar levels of education with comparable amounts having at least a high school diploma or less $(p=0.984)$. Both groups' level of income was equally distributed between each of the income quintiles $(p=0.744)$. There was no statistical significant difference in employment status between the two groups $(p=0.439)$; in both groups patients were predominantly retired or employed full-time. There was a statistically significant difference in marital status between the two groups with a lower proportion of patients within the marijuana group reported being married/common law ( $55.4 \%$ vs $63.2 \% ; p=0.048)$.

Tumor characteristics are summarized in Table 3 and statistical analysis represents comparison of proportions between the groups. The distribution of primary cancer sites was significantly different between marijuana users and the non-marijuana user group $(p<0.0001)$. There was a statistically significantly higher amount of oropharynx cancer within the marijuana user group (63.5\% vs $19.9 \%$, $p<0.0001$ ). As well, a higher percentage of the marijuana group's oropharyngeal cancers were found to be 16 positive $(95.7 \%$ vs $82.5 \%, p=0.002)$. Although the overall distribution of primary cancer sites were statistically different $(p<0.0001)$, similar rates of malignancy were observed between the marijuana and non-marijuana groups at the oral cavity ( $18.9 \%$ vs $17.5 \%)$, hypopharynx (1.4\% vs $1.5 \%)$, and larynx (16.2\% vs $17.2 \%)$. Even though no statistically significant differences were observed for the cT stage $(p=$ $0.076)$ and $\mathrm{cN}$ stage $(p=0.144)$, treatment modality differed significantly between the two groups $(p<0.0001)$. Marijuana patients were more likely to receive chemoradiation $(48.6 \%$ vs $24.2 \%)$ and less likely to receive surgery alone $(10.8 \%$ vs $20.8 \%)$ as compared to non-marijuana users $(p<0.0001)$.
Table 2 Socioeconomic Characteristics

\begin{tabular}{|c|c|c|c|}
\hline Variable & Non-Marijuana & Marijuana & $p$-Value \\
\hline $\mathrm{N}$ & 805 & 74 & \\
\hline Education Level, n (\%) & & & 0.984 \\
\hline Up to Grade 11 & $246(30.6)$ & $20(27.0)$ & \\
\hline High School Diploma & $226(28.1)$ & $23(31.1)$ & \\
\hline Trade School & $33(4.1)$ & $1(1.4)$ & \\
\hline College Diploma & $146(18.1)$ & $19(25.7)$ & \\
\hline University Diploma & $110(13.7)$ & $10(13.5)$ & \\
\hline Professional Degree & $5(0.6)$ & $1(1.4)$ & \\
\hline Master's Degree & $25(3.1)$ & $0(0)$ & \\
\hline $\mathrm{PhD}$ & $7(0.9)$ & $0(0)$ & \\
\hline Marital Status, n (\%) & & & 0.048 \\
\hline Married/Common Law & $509(63.2)$ & $41(55.4)$ & \\
\hline Single & $104(12.9)$ & $13(17.6)$ & \\
\hline Relationship & $3(0.4)$ & $2(2.7)$ & \\
\hline Divorced & $87(10.8)$ & $15(20.3)$ & \\
\hline Widowed & $102(12.7)$ & $3(4.1)$ & \\
\hline Income Quintile, n (\%) & & & 0.744 \\
\hline 1 & 159 (19.8) & $15(20.3)$ & \\
\hline 2 & $172(21.4)$ & $15(20.3)$ & \\
\hline 3 & $163(20.2)$ & $15(20.3)$ & \\
\hline 4 & $163(20.2)$ & $15(20.3)$ & \\
\hline 5 & $148(18.4)$ & $14(19.0)$ & \\
\hline Employment Status, n (\%) & & & 0.439 \\
\hline Full Time & $288(35.8)$ & $25(33.8)$ & \\
\hline Part Time & $27(3.3)$ & $1(1.4)$ & \\
\hline Retired & $399(49.6)$ & $35(47.3)$ & \\
\hline Unemployed & $40(5.0)$ & $3(4.1)$ & \\
\hline Disability & $44(5.5)$ & $10(13.5)$ & \\
\hline
\end{tabular}

\section{Discussion}

The first discussion about the demographics of marijuana use in HNC patients was by Donald in 1986 [17]. In a case series of six advanced head and neck squamous cell carcinoma cases, which were regular marijuana users, he commented on the exceptionally young age of the group, at a mean age of 27.1, as well as the fact that 2 out of 6 patients had never smoked tobacco [17]. This case series was the first to hint at a possible epidemiological variant in marijuana smoking HNCs. Since then case-control studies have examined the demographics of their respective cases and controls, although they have presented limited specific epidemiological data on the marijuana user cases.

The INHANCE pooled analysis combined five case control studies with $4029 \mathrm{HNC}$ cases from sites in United States and South America, but did not include 
Table 3 Tumor Characteristics

\begin{tabular}{|c|c|c|c|}
\hline Variable & Non-Marijuana & Marijuana & $p$-Value \\
\hline $\mathrm{N}$ & 805 & 74 & \\
\hline Primary Site of Cancer, n (\%) & & & $<0.0001$ \\
\hline Oropharynx & $160(19.9)$ & $47(63.5)$ & \\
\hline Oral Cavity & $141(17.5)$ & $14(18.9)$ & \\
\hline Nasopharynx & $19(2.4)$ & $0(0)$ & \\
\hline Hypopharynx & $12(1.5)$ & $1(1.4)$ & \\
\hline Larynx & $138(17.2)$ & $12(16.2)$ & \\
\hline Skin & $81(10.1)$ & $0(0)$ & \\
\hline Salivary Gland & $76(9.4)$ & $0(0)$ & \\
\hline Other & $177(22.0)$ & $0(0)$ & \\
\hline Oropharynx p16 Status & & & 0.002 \\
\hline P16 Positive & $132(82.5)$ & $45(95.7)$ & \\
\hline P16 Negative & $28(17.5)$ & $2(4.3)$ & \\
\hline cT-Stage, n (\%) & & & 0.076 \\
\hline Tis & $13(1.6)$ & $1(1.4)$ & \\
\hline $\mathrm{T} 1$ & $245(30.4)$ & $22(29.8)$ & \\
\hline $\mathrm{T} 2$ & $318(39.5)$ & $28(37.8)$ & \\
\hline T3 & $148(18.4)$ & $14(18.9)$ & \\
\hline T4 & $81(10.1)$ & $7(9.6)$ & \\
\hline cN-Stage, n (\%) & & & 0.144 \\
\hline NO & $357(44.4)$ & $31(43.0)$ & \\
\hline N1 & $100(12.4)$ & $8(11.1)$ & \\
\hline $\mathrm{N} 2 \mathrm{a}$ & $229(28.4)$ & $20(27.8)$ & \\
\hline $\mathrm{N} 2 \mathrm{~b}$ & $59(7.4)$ & $6(8.3)$ & \\
\hline $\mathrm{N} 2 \mathrm{C}$ & $30(3.7)$ & $3(4.2)$ & \\
\hline N3 & $30(3.7)$ & $4(5.6)$ & \\
\hline Treatment, n (\%) & & & $<0.0001$ \\
\hline S & 167 (20.8) & $8(10.8)$ & \\
\hline RT & $151(18.8)$ & $14(18.9)$ & \\
\hline CRT & $195(24.2)$ & $33(48.6)$ & \\
\hline S-RT & $197(24.4)$ & $12(16.2)$ & \\
\hline S-CRT & $84(10.4)$ & $6(8.1)$ & \\
\hline Palliative & $11(1.4)$ & $0(0)$ & \\
\hline
\end{tabular}

data from Canada [18]. The prevalence of marijuana use was found to be $10.1 \%$ amongst cases and there was a high proportion of males at $85.2 \%$, comparable to the $8.4 \%$ prevalence and $85.1 \%$ male percentage observed in our study. Marijuana users also similarly reported less tobacco and alcohol use compared to controls. The INHANCE study also found cancers at the oropharyngeal subsite most associated with marijuana, reflective of the subsite distribution observed in our data [18]. The population was more ethnically diverse compared to our study group with only $18.3 \%$ of $\mathrm{HNC}$ cases being
Caucasian, yet similar trends were observed [18]. A follow-up INHANCE analysis of an oropharyngeal subsite reiterated the relationship of this subsite with marijuana which increased with frequency and duration of use. Unfortunately, demographic and socioeconomic data was not compared between the marijuana users and non-users in the group. Marijuana's relationship with the oropharynx subsite was similarly found to be marginally associate with low smoking/alcohol use but potentially confounded by HPV exposure [16]. However, the primary goal of the INHANCE analyses were not to examine epidemiological variation and only limited comparisons can be made to our data with regards to p16 status, marriage, income, employment, clinical stage, and treatment regime as they were not examined.

Based on 2012 Canada census data, there are a few demographic patterns seen in marijuana users that are part of the general Canadian population [1]. They tend to be younger with $33 \%$ being $18-24$ and only $0.8 \%$ aged 65 or older [1]. There is a male predominance in consistent users with $4.6 \%$ of males vs $1.7 \%$ of females reporting weekly use and $2.4 \%$ of males and $1.2 \%$ of females reporting daily use [1]. Lifetime marijuana use and marijuana use within the past year were also higher in males compared to females at $49.4 \%$ vs 35.8 and $16.1 \%$ vs $8.3 \%$, respectively [1]. In contrast to the young age of marijuana smokers in the general population, our study's marijuana users were older with a mean age of 62.26 . Based on at least weekly marijuana usage, our study observed a 5.7:1 male predominance in the marijuana user group; this is higher than the male predominance observed in the general population both in weekly users (3:1) and daily users (2:1) [1]. The differences in gender and mean age between our population subset compared to that of Canada census data could be related to the epidemiology of HNC where the mean age often range from 55 to 65 years old depending on the disease site and are often predominantly male [19]. While Canada census data provides an overview of the general population, our cross sectional sample of HNC cannot be discounted.

The epidemiology of HNCs related to tobacco smoke and alcohol have also been well studied. Patients have been shown to have peak incidence in late-middle age at $55-59$ with a significant $5: 1$ male predominance $[20,21]$. The proportion of patients having an educational attainment higher than a high school diploma are similar between the tobacco and alcohol related HNCs at 29.2 and $31 \%$, respectively $[20,21]$. This is similar to patients within our non-marijuana user population where the mean age was 56.6 with a predominantly male population. Our study's marijuana group however, presented slightly later in late-middle age at 62.26 but with a similar 5.7:1 male predominance. Moreover, the marijuana group had higher educational attainment with $41.9 \%$ of 
patients having higher than a high school diploma. It is still unclear whether these subtle demographic variations within the marijuana user HNC population have any significant effect on the treatment or survivorship outcomes. Certainly more knowledge on this subgroup of patients is needed in the future and could provide interesting insights.

The only variation in socioeconomic characteristics identified from our data set was that HNC patients that reported marijuana use are less likely to be married/ common law compared to those that did not $(55.4 \%$ vs $63.2 \% ; p=0.048)$. Marital status has been shown to be an independent prognostic factor in HNCs; however, given the other differences in this population, the significance or impact of marital status among marijuana smoking HNC patients remains undetermined [22]. It is interesting that the marijuana user group were not only more likely to be single but also had higher rates of HPV positive oropharynx cancer, a factor associated with increased sexual practices and partners [15]. While quantification of the amount of partners within this subsite group of patients was beyond the scope of this study future studies is warranted to delineate any potential relationships. The remaining socioeconomic characteristics including educational attainment, income, and employment status were not different between the HNC patients in the marijuana and non-marijuana groups.

HPV positive oropharyngeal cancer was the site of the highest prevalence in the marijuana user group compared to the non-user group (oropharyngeal cancer $63.5 \%$ vs $19.9 \%, p<0.0001$; p16 positive $95.7 \%$ vs $82.5 \%$; $p=0.002$ ). This is reflective of the shift to HPV positive oropharynx cancers as the predominant head and neck disease site in Ontario and North America [23-25]. The difference in treatment modality between the two groups supports this as the marijuana user group had statistically higher chemoradiation $(p<0.0001)$ as the primary therapy, which is the standard treatment option for this disease site within our cancer center. Interestingly, patients who were marijuana users were also found to have statistically lower incidence of tobacco use $(p=0.001)$. This coupled with the higher incidence of HPV positive oropharynx cancer within the marijuana user group suggest that patients with recreational marijuana use are reflective of the trends in epidemiological variation of HNC patients. These variations could suggest differences in sexual practices between marijuana users and nonusers, the potential direct oncogenic effects of marijuana use, or something entirely different that is not yet defined. While the establishment of a true cause and effect relationship is beyond the scope of this study, it is intriguing and would certainly warrant further research.

This is a population-based study with prospectively collected data but is subject to limitations. The primary limitation of this study is the small sample size of HNC patients that reported marijuana use. We were also unable to sub-stratify the marijuana user group based on the quantity of use. Unlike smoking and alcohol, marijuana has not been concretely established as a risk factor for $\mathrm{HNC}$ and there is no validated clinically significant cut-off for marijuana frequency/use. We elected to use the definition of at least weekly use based on extrapolation of findings in the literature. Within the setting of $\mathrm{HNC}$, there is data to show marijuana use at a frequency of less than three times per week or at least once monthly is associated with oropharyngeal and HPV-related cancers [15, 16]. Beyond oncology, at least weekly cannabis use has been found to be predictive of adverse events in the context of psychosis, neuropsychological function, and stroke/TIA [26-29]. In addition, since cannabis remains classified as Schedule II substance under the Canadian Controlled Drugs and Substances Act, there may be an under-reporting of marijuana use in the study population which may affect the subsequent break down of patient as well as socioeconomic characteristics of patients [30]. Patient income quintiles were extrapolated from neighbourhood level income and thus the interpretation may be susceptible to ecological fallacy. Despite potential discordance, neighbourhood and individual level income have been shown to produce comparable observations [31, 32]. Due to the inadequate follow-up data, we also were not able to discover the potential effects of marijuana on HNC patients. Future long-t erm prospectively based studies would help mitigate and answer more questions on the relationship of marijuana on HNC patients.

To our knowledge this is the first study to look at the epidemiological variances within HNC patients who are marijuana users. Patients were found to have predominantly HPV positive oropharynx cancer and more likely to be single with statistically significant less tobacco use. There was no statistically significant difference between the two groups in cT and cN Stage as well as age at diagnosis, alcohol use, Karnofsky score, education level, ethnicity, employment status, and income quintiles. This study has also highlighted variations in epidemiology compared to marijuana users in general and HNC patients that smoke and use alcohol.

\section{Conclusion}

Patients diagnosed with HNC who are marijuana users do present with subtle variation in tumor and socioeconomic characteristics when compared with non-marijuana users. This study provides an overview of these epidemiological variations and will be a useful adjunct for future studies within this area that explore the potential oncologic as well as quality of life of life effects marijuana has on HNC patients. 


\section{Additional file}

Additional file 1: Juravinski Cancer Center Head and Neck Cancer Intake Database. (DOCX 15 kb)

\section{Acknowledgements}

Not applicable.

NB: The corresponding author has full access to the data in this study and takes responsibility for the integrity of the data and accuracy of the data analysis.

\section{Funding}

The authors declare no relevant sources of funding.

\section{Availability of data and materials}

The datasets used and/or analysed during the current study are available from the corresponding author on reasonable request.

\section{Authors' contributions}

MX analyzed and interpreted the data and was a major contributor in writing the manuscript. $\mathrm{HZ}$ was involved in the conception and design, acquired, analyzed, and interpreted the data and was a major contributor in writing the manuscript. SA was involved in conception and design, acquisition of data, and interpretation of data. BSJ was involved in conception and design, acquisition of data, and interpretation of data. JEMY was involved in conception and design, acquisition of data, and interpretation of data. MKG was involved in the conception and design, acquired and interpreted the data and was a major contributor in reviewing the manuscript. All authors read and approved the final manuscript

\section{Ethics approval}

This study was approved by the Hamilton Integrated Research Ethics Board. Consent for study participation was obtained from patients recruited to participate in the study.

\section{Consent for publication}

Not applicable.

\section{Competing interests}

The authors declare that they have no competing interests.

\section{Publisher's Note}

Springer Nature remains neutral with regard to jurisdictional claims in published maps and institutional affiliations.

\section{Author details}

${ }^{1}$ Division of Otolaryngology-Head and Neck Surgery, McMaster University, G811, 50 Charlton Avenue E, Hamilton, ON L8N 4A6, Canada. ${ }^{2}$ Michael G. DeGroote School of Medicine, McMaster University, Hamilton, ON, Canada.

Received: 4 September 2018 Accepted: 6 November 2018

Published online: 22 November 2018

\section{References}

1. Rotermann M, Langlois K. Prevalence and correlates of marijuana use in Canada, 2012. Heal Rep. 2015;26(4):10-5.

2. Cerdá M, Wall M, Keyes KM, Galea S, Hasin D. Medical marijuana laws in 50 states: investigating the relationship between state legalization of medical marijuana and marijuana use, abuse and dependence. Drug Alcohol Depend. 2012;120(1-3):22-7.

3. Jones J, Nicole Jones K, Peil J. The impact of the legalization of recreational marijuana on college students. Addict Behav. 2018;77:255-9.

4. Joseph LA, Routledge JA, Burns MP, et al. Value of the hospital anxiety and depression scale in the follow up of head and neck cancer patients. J Laryngol Otol. 2013;127(03):285-94.

5. Hassanein KA-AM, Musgrove BT, Bradbury E. Psychological outcome of patients following treatment of oral cancer and its relation with functional status and coping mechanisms. J Cranio-Maxillofacial Surg. 2005;33(6):404-9.
6. Gil F, Costa G, Hilker I, Benito L. First anxiety, afterwards depression: psychological distress in Cancer patients at diagnosis and after medical treatment. Stress Heal. 2012;28(5):362-7.

7. Nguyen NP, Vos P, Karlsson U, et al. Quality of life following Chemoradiation and postoperative radiation for locally advanced head and neck Cancer. ORL. 2007:69(5):271-6.

8. Gold D. The psychosocial care needs of patients with HPV-related head and neck Cancer. Otolaryngol Clin N Am. 2012;45(4):879-97.

9. Osborne $\mathrm{GB}$, Fogel C. Understanding the motivations for recreational marijuana use among adult Canadians. Subst Use Misuse. 2008;43(3-4): 539-72.

10. Elliott DA, Nabavizadeh N, Romer JL, Chen Y, Holland JM. Medical marijuana use in head and neck squamous cell carcinoma patients treated with radiotherapy. Support Care Cancer. 2016;24(8):3517-24.

11. Roby TJ, Hubbard G, Swan GE. Cytomorphologic features of sputum samples from marijuana smokers. Diagn Cytopathol. 1991;7(3):229-34.

12. Sarafian TA, Magallanes JAM, Shau H, Tashkin D, Roth MD. Oxidative stress produced by marijuana smoke. Am J Respir Cell Mol Biol. 1999; 20(6):1286-93.

13. Barsky SH, Roth MD, Kleerup EC, Simmons M, Tashkin DP. Histopathologic and molecular alterations in bronchial epithelium in habitual smokers of marijuana, cocaine, and/or tobacco. JNCI J Natl Cancer Inst. 1998;90(16): 1198-205.

14. Huang Y-HJ, Zhang Z-F, Tashkin DP, Feng B, Straif K, Hashibe M. An epidemiologic review of marijuana and cancer: an update. Cancer Epidemiol Biomark Prev. 2015;24(1):15-31.

15. Gillison ML, D'Souza G, Westra W, et al. Distinct risk factor profiles for human papillomavirus type 16-positive and human papillomavirus type 16-negative head and neck cancers. JNCI J Natl Cancer Inst. 2008; 100(6):407-20.

16. Marks MA, Chaturvedi AK, Kelsey K, et al. Association of Marijuana Smoking with oropharyngeal and Oral tonque cancers: pooled analysis from the INHANCE consortium. Cancer Epidemiol Biomark Prev. 2014; 23(1):160-71.

17. Donald PJ. Marijuana smoking - possible cause of head and neck carcinoma in young patients. Otolaryngol Neck Surg. 1986;94(4):517-21.

18. Berthiller J, Lee Y-CA, Boffetta P, et al. Marijuana smoking and the risk of head and neck cancer: pooled analysis in the INHANCE consortium. Cancer Epidemiol Biomark Prev. 2009;18(5):1544-51.

19. Rettig EM, D'Souza G. Epidemiology of head and neck Cancer. Surg Oncol Clin N Am. 2015;24(3):379-96.

20. Wyss A, Hashibe M, Chuang S-C, et al. Cigarette, cigar, and pipe smoking and the risk of head and neck cancers: pooled analysis in the international head and neck Cancer epidemiology consortium. Am J Epidemiol. 2013; 178(5):679-90.

21. Marron M, Boffetta P, Zhang Z-F, et al. Cessation of alcohol drinking, tobacco smoking and the reversal of head and neck cancer risk. Int J Epidemiol. 2010;39(1):182-96.

22. Inverso G, Mahal BA, Aizer AA, Donoff RB, Chau NG, Haddad RI. Marital status and head and neck cancer outcomes. Cancer. 2015;121(8):1273-8.

23. Johnson-Obaseki S, McDonald JT, Corsten M, Rourke R. Head and neck Cancer in Canada. Otolaryngol Neck Surg. 2012;147(1):74-8.

24. Mifsud M, Eskander A, Irish J, et al. Evolving trends in head and neck cancer epidemiology: Ontario, Canada 1993-2010. Head Neck. 2017;39(9):1770-8.

25. Chaturvedi AK, Engels EA, Pfeiffer RM, et al. Human papillomavirus and rising oropharyngeal Cancer incidence in the United States. J Clin Oncol. 2011:29(32):4294-301.

26. Bechtold J, Hipwell A, Lewis DA, Loeber R, Pardini D. Concurrent and sustained cumulative effects of adolescent marijuana use on subclinical psychotic symptoms. Am J Psychiatry. 2016;173(8):781-9.

27. Degenhardt $L$, Hall W. The association between psychosis and problematical drug use among Australian adults: findings from the National Survey of mental health and well-being. Psychol Med. 2001;31(4):659-68.

28. Meier $\mathrm{MH}$, Caspi A, Ambler A, et al. Persistent cannabis users show neuropsychological decline from childhood to midlife. Proc Natl Acad Sci U S A. 2012;109(40):E2657-64.

29. Hemachandra D, McKetin R, Cherbuin N, Anstey KJ. Heavy cannabis users at elevated risk of stroke: evidence from a general population survey. Aust N Z J Public Health. 2016;40(3):226-30.

30. Government of Canada. Controlled Drugs and Substances Act. Ottawa: Government of Canada; 2016. 
31. Pichora E, Polsky JY, Catley C, Perumal N, Jin J, Allin S. Comparing individual and area-based income measures: impact on analysis of inequality in smoking, obesity, and diabetes rates in Canadians 2003-2013. Can J Public Heal. 2018;109(3):410-8.

32. Southern DA, McLaren L, Hawe P, Knudtson ML, Ghali WA. Individual-Level and Neighborhood-Level Income Measures: Agreement and Association with Outcomes in a Cardiac Disease Cohort. Med Care. 2005;43:1116-22.

Ready to submit your research? Choose BMC and benefit from:

- fast, convenient online submission

- thorough peer review by experienced researchers in your field

- rapid publication on acceptance

- support for research data, including large and complex data types

- gold Open Access which fosters wider collaboration and increased citations

- maximum visibility for your research: over $100 \mathrm{M}$ website views per year

At BMC, research is always in progress.

Learn more biomedcentral.com/submissions 